\title{
Introduction to Extracellular Vesicles: Biogenesis, RNA Cargo Selection, Content, Release, and Uptake
}

\author{
Erik R. Abels ${ }^{1,2} \cdot$ Xandra O. Breakefield ${ }^{1}$
}

Received: 8 September 2015/Accepted: 21 March 2016/Published online: 6 April 2016

(C) Springer Science+Business Media New York 2016

\begin{abstract}
Extracellular vesicles are a heterogeneous group of membrane-limited vesicles loaded with various proteins, lipids, and nucleic acids. Release of extracellular vesicles from its cell of origin occurs either through the outward budding of the plasma membrane or through the inward budding of the endosomal membrane, resulting in the formation of multivesicular bodies, which release vesicles upon fusion with the plasma membrane. The release of vesicles can facilitate intercellular communication by contact with or by internalization of contents, either by fusion with the plasma membrane or by endocytosis into "recipient" cells. Although the interest in extracellular vesicle research is increasing, there are still no real standards in place to separate or classify the different types of vesicles. This review provides an introduction into this expanding and complex field of research focusing on the biogenesis, nucleic acid cargo loading, content, release, and uptake of extracellular vesicles.
\end{abstract}

Keywords Extracellular vesicles - Exosomes ·

Microvesicles · Biogenesis · RNA · Cargo selection

Erik R. Abels

eabels@mgh.harvard.edu

1 Departments of Neurology and Radiology, Massachusetts General Hospital and NeuroDiscovery Center, Harvard Medical School, Boston, MA 02114, USA

2 Department of Neurosurgery, Neuro-Oncology Research Group, VU University Medical Center, 1007MB Amsterdam, The Netherlands

\section{Introduction}

Extracellular vesicles (EVs) are a heterogeneous family of membrane-limited vesicles originating from the endosome or plasma membrane. Pan and Johnstone (1983) were among the first to describe EVs. Initially, it was shown that the release of EVs was part of a disposal mechanism to discard unwanted materials from cells. Subsequent research has shown that the release of EVs is also an important mediator of intercellular communication that is involved in normal physiological process as well as in pathological progression (Frühbeis et al. 2012, 2013; Marcilla et al. 2012; Luga et al. 2012; Regev-Rudzki et al. 2013; Barteneva et al. 2013).

EVs are currently classified based on their mode of release or size. EVs can be released by "donor" cells either through the outward budding of the plasma membrane, termed shedding microvesicles (MVs) or ectosomes (Minciacchi et al. 2015). Another release process involves the inward budding of the endosomal membrane, resulting in the formation of multivesicular bodies (MVBs), with exosomes released by fusion of the outer MVB membrane to the plasma membrane (Théry et al. 2009; Denzer et al. 2000). Vesicles may also be released from nanotubular structures extending from the plasma membrane (Rilla et al. 2013, 2014). In addition to the differences in the mode of release, the size of the vesicles is also used for characterization. Although different scales are used, MVs range from 50 to $10,000 \mathrm{~nm}$, and exosomes are smaller with a diameter of 30 to $150 \mathrm{~nm}$ (György et al. 2011; Baietti et al. 2012; Colombo et al. 2013). Overall EVs comprise a wide variety of vesicles ranging from 30 to $1000 \mathrm{~nm}$ in size with a variety of cargos, and the different types of vesicles overlap in their size distribution. It must be emphasized that there is some controversy on 
nomenclature and sizes of the different types of vesicles (Gould and Raposo 2013; Witwer et al. 2013); however, basic requirements of criteria for EVs have been established (Lötvall et al. 2014). So far no real standards have been set to classify the different types of vesicles, so one should be careful with the use of size alone in defining different types of vesicles. In the future the mode of biogenesis, means of isolation and cargo may turn out to be far more important criteria. Given how the different isolation methods may influence the nature of EVs, methods should be compared in order to develop a gold standard for the different protocols and measurements (Momen-Heravi et al. 2012). To be able to compare results, it must be stressed that publications on EVs need to clarify their isolation methods in detail, and in general term, EVs should be used unless there are specific markers defined to classify the different types of vesicles.

So far, extensive evidence on all these different types of vesicles indicates that EVs are a key player in the intercellular communication between cells, along with secretion of small soluble molecules (the secretome) and cell-cell contact (Raposo and Stoorvogel 2013; Cocucci et al. 2009). Once released the EVs can be internalized via endocytosis or membrane fusion, releasing their contents into "recipient" cells (Mulcahy et al. 2014). Recent studies have shown that these EVs contain various proteins, sugars, lipids, and a wide variety of genetic materials, such as DNA, mRNA, and non-coding (nc)RNAs with the content protected from proteases and nucleases in the extracellular space by the limiting membrane (Henderson and Azorsa 2012; Théry et al. 2002). EVs have the potential to deliver combinatorial information to multiple cells in their tissue microenvironment and throughout the body (Baj-Krzyworzeka et al. 2006; Ratajczak et al. 2006; Skog et al. 2008).

This review provides an introduction into the world of EVs, focusing primarily subtypes labeled as exosomes and MVs, and discusses basics of the biogenesis, nucleic acid cargo loading, content, release, and uptake of these vesicles. Thus, it provides the necessary background for interpretation of the articles in this Special Issue on the role of EVs in the neurobiology and diseases of the nervous system.

\section{The Ins and Outs of EVs}

\section{Vesicle Biogenesis}

As EVs have traditionally been classified based on differences in biogenesis, we will focus on the different molecular mechanisms resulting in either the release of vesicles upon the fusion of the MVBs with the plasma membrane or the release via the outward budding and fission of the plasma membrane (Akers et al. 2013).

\section{Exosome Biogenesis}

Exosomes are derived from the endosomal system, and are formed as intraluminal vesicles (ILVs) in the MVBs. This network of ILVs is used to degrade, recycle or exocytose proteins, lipids, and nucleic acids. Within the endosomal system or endocytic pathway, the endosomes are divided into different compartments-early endosomes, late endosomes, and recycling endosomes (Grant and Donaldson 2009). Endosomes form by invagination of the plasma membrane. The early endosomes can fuse with endocytic vesicles, at which point the content is destined for degradation, recycling or secretion. Contents to be recycled are sorted into recycling endosomes (Morelli et al. 2004). The remaining early endosomes transform into late endosomes (Stoorvogel et al. 1991). The late endosomes accumulate ILVs formed by inward budding of the endosomal membrane. During this process, cytosolic proteins, nucleic acids, and lipids are sorted into these small vesicles. Late endosomes containing a multitude of small vesicles are termed MVBs. These MVBs can either fuse with the lysosome if the content is fated for degradation or fuse with the cellular membrane releasing the ILVs as exosomes into the extracellular space (Grant and Donaldson 2009).

The formation of the ILVs within MVBs is the start of the biogenesis of exosomes. ILV formation requires two distinct processes. First, the endosome membrane is reorganized such that it becomes highly enriched for tetraspanins (Pols and Klumperman 2009). The two tetraspanins that are thought to play a critical role in exosome formation are CD9 and CD63. Second, the endosomal sorting complexes required for transport (ESCRTs) are recruited to the site of ILV formation (Wollert and Hurley 2010; Colombo et al. 2013). Four different ESCRTs have been identified, ESCRT 0, I, II, and III (Henne et al. 2011). ESCRT 0 recognizes ubiquitinated proteins on the outside of the endosomal membrane (Raiborg and Stenmark 2009). ESCRT I and II are recruited to cytosolic side of the early endosomes via various stimuli. For example, ESCRT recruitment is stimulated by the presence of phosphatidylinositol 3-phosphate (PIP3), the hepatocyte growth factor-regulated tyrosine kinase substrate (HRS), the ubiquitination of the cytosolic tail of endocytosed proteins and/or the curved membrane topology (Tamai et al. 2010; Shields et al. 2009; Razi and Futter 2006; Katzmann et al. 2001; Bache et al. 2003; Fernandez-Borja et al. 1999). It has been suggested that ESCRT I and II are the initiators and drivers of the intraluminal membrane budding, whereas ESCRT III completes this process (Babst et al. 2002; Henne et al. 2013). In short, ESCRT I binds the 
ubiquitinated cargo on the endosomes, upon which ESCRT II is activated (Katzmann et al. 2001). In turn, ESCRT III is recruited through programmed cell death 6 interacting protein (PDCD6IP or ALIX), and associates with the tumor susceptibility gene 101 (TSG101) as part of the ESCRT I complex (Matsuo et al. 2004; Baietti et al. 2012). To conclude, ALIX serves as intermediate between the association between ESCRT I and ESCRT III, as it binds the TSG101 component of ESCRT I and the charged MVB protein 4A (CHMP4A) components of ESCRT III (McCullough et al. 2008). This process is finalized through the sequestration of MVB proteins and recruitment of a deubiquitinating enzyme, which removes the ubiquitin tag from the cargo proteins prior to sorting them into the ILVs. Finally, ESCRT-III is disassembled for recycling by AAAATPase suppressor-of-potassium-transport-growth-defect1 protein (SKD1) (Bishop and Woodman 2001; Benedetto et al. 2006) (Fig. 1a).

Recent evidence has revealed an alternative ESCRT pathway, the syndecan-syntenin-ALIX pathway, where heparanase, syndecan heparan sulfate proteoglycans, ADP ribosylation factor 6 (ARF6), phospholipase D2 (PLD2), and syntenin mediate exosome biogenesis, including vesicle formation and loading of proteins (Baietti et al. 2012). The formation of ILVs is facilitated by the interaction of syntenin with ALIX and depends on the availability of heparan sulfate, syndecans, ALIX, and ESCRTs (Baietti et al. 2012). Heparanase stimulates release of exosomes containing syntenin-1, syndecan, and CD63 and facilitates endosomal membrane budding and the biogenesis of exosomes by enzymatic digestion of heparan sulfate chains on syndecans (Roucourt et al. 2015). This mode of ILV

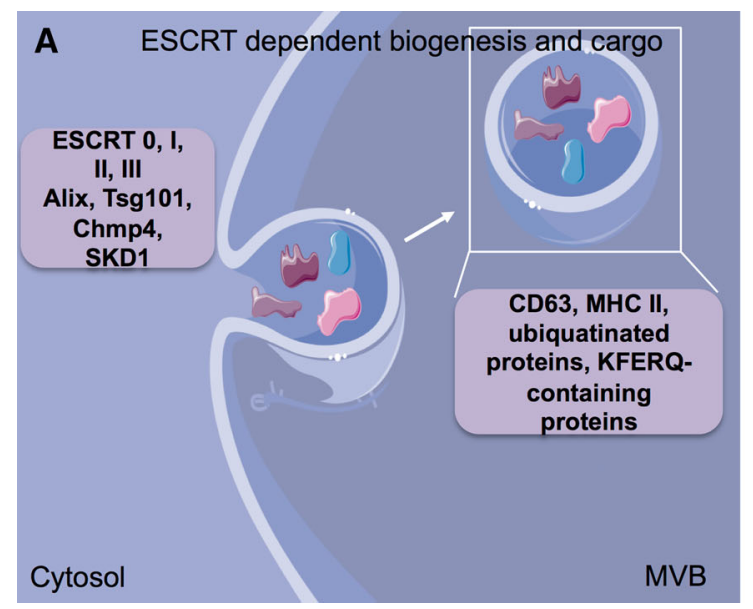

Fig. 1 Molecular mechanisms of ESCRT-dependent and -independent MVB biogenesis. Multiple biogenesis machineries have been described for generating ILVs in MVBs. a ESCRT-dependent MVB biogenesis requires the ESCRT protein and ESCRT-associated proteins (ALIX, TSG101, Chmp4, and SKD1) to form MVBs containing CD63, MHC II, ubiquitinated proteins and KFERQ- budding is controlled by ARF6 and PLD2 (Ghossoub et al. 2014) (Fig. 1b).

Although the ESCRT pathway is generally thought to be the main driver of exosomal biogenesis, different studies have shown the existence of ESCRT-independent exosome biogenesis. For example, inactivation of the ESCRTs does not inhibit the formation of MVBs (Stuffers et al. 2009). Other mechanisms of exosome biogenesis could operate in parallel to the ESCRT pathway and vary depending on the cell type and vesicle content. Trajkovic and colleagues (2008) found that the formation of ILVs through the inward budding of the limiting membrane of the MVBs required sphingolipid ceramide. This lipid could facilitate the membrane invagination of ILVs through its cone-shaped structure. The release of exosomes could even be reduced after the inhibition of neutral sphingomyelinase (nSMase), a protein responsible for the production of ceramide. Adding to this observation is the abundance of ceramide and its derivatives in exosomes, as well as the presence of proteolipoprotein (PLP), CD63, CD81, and TSG101 (Wubbolts et al. 2003; Brouwers et al. 2013). However, in some cell types, e.g., human melanoma cells depletion of nSMase does not inhibit the formation of MVB or exosome release (Fig. 1b). To summarize, the difference in exosome content relates, at least in part, to the various machineries involved in exosome biogenesis (Fig. 1).

\section{Exosome Release}

Release of exosomes into the extracellular space is facilitated by the fusion of the MVB limiting membrane with the plasma membrane. Similar to the different mechanisms

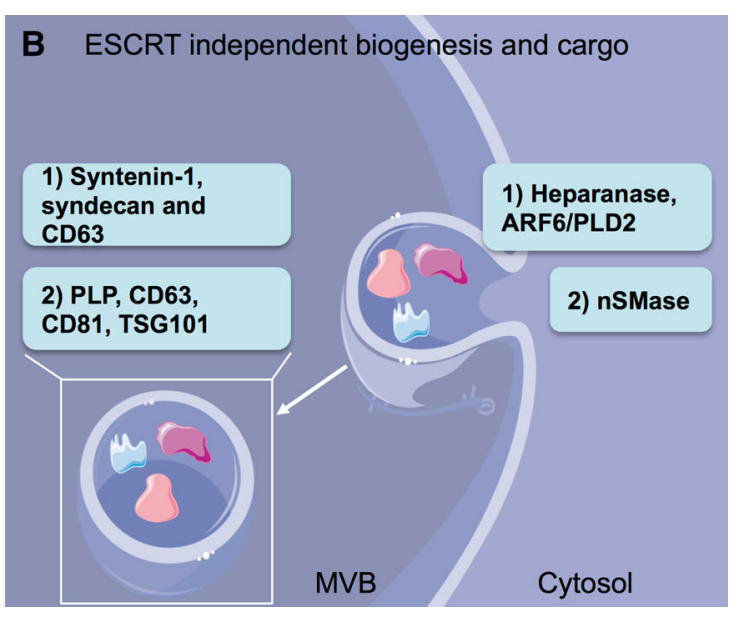

containing proteins. b Three ESCRT-independent pathways are controlled by different proteins: 1 heparanase and ARF6/PLD2, associated with the presence of syntenin-1, syndecan, and CD63 in exosomes; 2 nSMase, in which the exosomes are enriched with PLP, CD63, CD81, and TSG101 [Components in image derived from Servier Medical Art Powerpoint image bank (Servier 2016)] 
proposed for the biogenesis of exosomes, a variety of mechanisms have also been proposed for the release of exosomes. A number of Rab GTPases, including RAB11 and $\mathrm{RAB} 35$, or RAB27A and $\mathrm{RAB} 27 \mathrm{~B}$, are recognized to play an important role. Release of exosomes through fusion of the MVB with the plasma membrane facilitated by RAB11 and RAB35 was first found when screening with dominate-negative Rab GTPase mutants (Savina et al. 2003; Hsu et al. 2010). The exosomes release via this mechanism are enriched in proteins, such as flotillin and other cell-specific proteins, including Wnt, PLP, and the transferrin receptor (TfR) (Laulagnier et al. 2004) (Fig. 2a). The exosomes released via RAB27A/B are linked to late endosomal and secretory compartments and are enriched in late endosomal proteins (e.g., CD63, ALIX, and TSG101) (Stenmark 2009; Ostrowski et al. 2010). Interestingly, a different Rab GTPase, RAB7, is involved in the release of exosomes containing ALIX and syntenin by breast tumor cells (Baietti et al. 2012), although depletion of RAB7 does not affect exosome release in

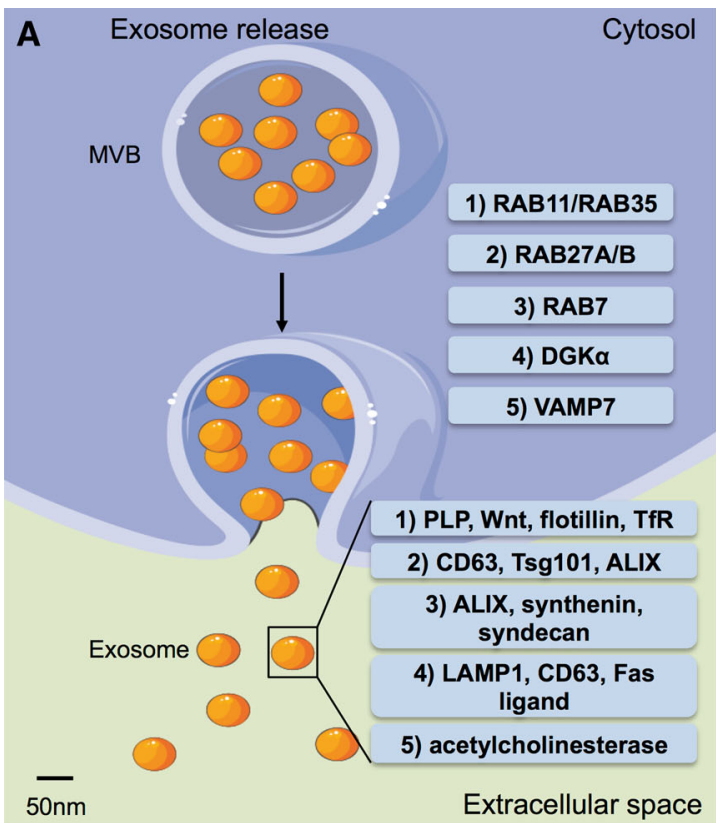

Fig. 2 Molecular machineries of EV release. a Proteins involved in controlling the fusion of MVBs with the outer membrane to the plasma membrane, resulting in release of exosomes. Five different machineries have been described so far; 1 RAB11 and RAB35 facilitate the fusion of MVBs to the plasma membrane, releasing exosomes containing PLP, Wnt, flotillin, and TfR; 2 RAB27A and RAB27B promote release of exosomes loaded with CD63, TSG101, and ALIX; 3 RAB7-dependent release yields release of exosomes harboring ALIX, synthenin, and syndecan; $4 \mathrm{DGK} \alpha$ protein is implicated in release of exosomes carrying LAMP1, CD63, and Fas ligand; and 5 VAMP7 regulates the membrane fusion associated with release of acetylcholinesterase-containing exosomes release. b EV some other cells, e.g., HeLa cells (Ostrowski et al. 2010). In addition, some exosomes may be released through budding from the plasma membrane independently of Rab GTPases. For example, diacylglycerol kinase $\alpha$ (DGK $\alpha)$ has been shown to negatively control release of LAMP1/ CD63 positive exosomes containing Fas-ligands (Alonso et al. 2007). But since DGK $\alpha$ is a negative regulator of MVB formation, the inhibition of exosome release could also result from decreased exosome generation (Alonso et al. 2011, 2007). SNARE proteins have been implicated in the membrane fusion of two organelles (Rao et al. 2004; Logan et al. 2006; Puri and Roche 2008; Tiwari et al. 2008). One of the SNARE proteins, the vesicle-associated membrane protein 7 (VAMP7), has been described to stimulate the release of acetylcholinesterase-containing exosomes (Fader et al. 2009). To conclude, exosomes can be generated and released from different subtypes of endosomes by various mechanisms and harbor different cargos as a function of cell type and probably physiologic state (Fig. 2a).

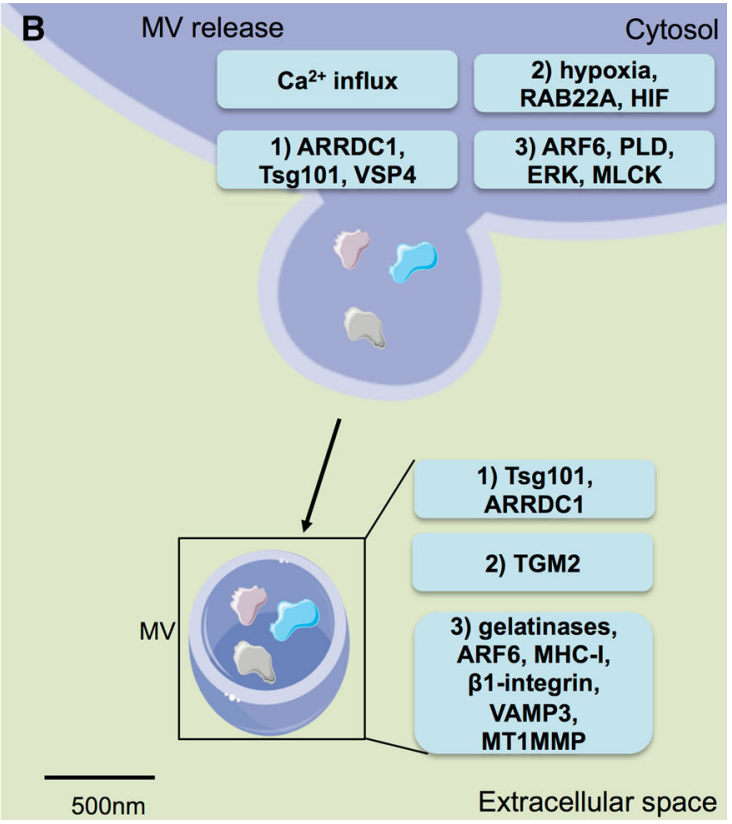

released via the outward budding and fission of the plasma membrane controlled by different proteins and extracellular signaling results in release of MVs with a distinct protein profile. Three pathways have been described including markers found in released MVs: a ARRDC1, TSG101, and VSP4 are responsible for the shedding of MVs containing TSG101 and ARRDC1; b hypoxia following expression of RAB22A via HIF, characterizes the secretion of EVs carrying TGM2; and $\mathbf{c}$ the ARF6, PLD, ERK, and MLCK cascade induces release of EVs containing gelatinases, ARF6, MHC-I, $\beta 1$ integrin, VAMP3, and MT1MMP. [Components in image derived from Servier Medical Art Powerpoint image bank (Servier 2016)] 


\section{Microvesicle Biogenesis and Release}

The biogenesis of the MVs is far less defined as compared to exosomes. Biogenesis and release of MVs has been investigated in several cellular model systems. Different mechanisms are found to be responsible for the shedding of MVs. In general, these types of vesicles appear to be formed though the outward budding and fission of the plasma membrane (Fig. 2b). A combination of factors will result in the formation of MVs such as the redistribution of phospholipids, including the repositioning of phosphatidylserine to the outer leaflet, and contraction of the actin-myosin machinery (Akers et al. 2013). In detail, ADP-ribosylation factor 6 (ARF6) initiates a cascade that activates phospholipase D (PLD). Next, the extracellular signal-regulated kinase (ERK) is recruited to the plasma membrane, where it phosphorylates and activates the myosin light chain kinase (MLCK). Finally, the phosphorylation and activation of the myosin light chain by MLCK trigger the release of the MVs. These MVs have been described as being specifically loaded with ARF6, MHC-I, $\beta 1$-integrin, VAMP3, and MT1MMP (Muralidharan-Chari et al. 2009). Interestingly, a recent study provided evidence for the recruitment of the ESCRT-I subunit TSG101 to the plasma membrane through its binding to a tetrapeptide protein within the Arrestin 1 domain-containing protein 1 (ARRDC1), resulting in the release of MVs containing TSG101, ARRDC1, and other cellular proteins (Nabhan et al. 2012; Tauro et al. 2012). The formation of these MVs required VPS4 ATPase with E3 ligase WWP2 interacting and ubiquitinating ARRDC1 (Nabhan et al. 2012). Furthermore, external factors can induce MV release. For example, the influx of calcium induces the redistribution of the phospholipids resulting in increased release of MVs (Bucki et al. 1998; Pasquet et al. 1996). In addition, hypoxia is been shown to promote MV release via HIFdependent expression of RAB22A (Wang et al. 2014). Again, the different mechanisms underlining the release of MV from the plasma membrane can be distinguished based on the content of the released MVs (Fig. 2b). Some of these mechanisms are similar to those described for extracellular budding of virus particles, such as retroviruses (Gould et al. 2003), and, in fact, a substantial portion of EVs released from cancer cells are retrovirus-like particles (Akers et al. 2013; Balaj et al. 2011).

\section{Contents of the Different Types of Vesicles}

The contents of vesicles vary with respect to mode of biogenesis, cell type, and physiologic conditions. In general, all EVs are loaded with various proteins, lipids, and nucleic acids. The loading of the different types of cargo can be specific per vesicle and cell type. Extensive research has been carried out to characterize the content of EVs. This has resulted in the assembly of different databases collecting the datasets from the many EV studies. Three different databases are publicly accessible: Exocarta, Vesiclepedia, and EVpedia (Kim et al. 2013; Kalra et al. 2012; Mathivanan and Simpson 2009; Simpson et al. 2012; Mathivanan et al. 2012). All databases include the protein, nucleic acid, and lipid content together with the isolation and purification procedures used to generate the data. Here we give a broad overview of various cargos within EVs, with an emphasis on the nucleic acid content.

\section{Protein Content}

Comprehensive research has been done on the protein cargo of EVs, profiling the contents of different-sized vesicles produced by various cell types (Conde-Vancells et al. 2008; Demory Beckler et al. 2013; Gonzalez-Begne et al. 2009; Graner et al. 2009; Théry et al. 2001; Turiák et al. 2011). However, due to the variations in isolation techniques and the different cell types and culture conditions used to analyze the protein content, it is difficult to give a conclusive view of the protein composition of the different types of vesicles. Commonly found proteins in EVs are those associated with the mechanisms responsible for biogenesis, including proteins associated with the endosomal pathway. For example, components of the ESCRTs are enriched in the vesicle fraction, e.g., ALIX, TSG101. Additionally, proteins responsible for EV formation and release, such as RAB27A, RAB11B, and ARF6, are also commonly found. Moreover, EVs contain different types of tetraspanins, including CD63, CD81, and $\mathrm{CD} 9$, as well as proteins involved in signal transduction (EGFR), antigen presentation (MHC I and MHC II) and other transmembrane proteins (LAMP1, TfR). In general, proteins associated with the endoplasmic reticulum, Golgi, and nucleus are not found in EVs (Théry et al. 2001), but there have been reports of transcription factors inside EVs, e.g., Notch, Wnt, which are normally found in the nucleus (Kalra et al. 2012). Some research has gone into discovering principles of how proteins can be loaded into vesicles, which involves association with the plasma membrane as an oligomeric complex (Yang and Gould 2013). As mentioned before, the discrepancies between the different datasets and the different techniques used to analyze the content of the vesicles calls for standardization of isolation and analysis techniques to clarify the protein composition of the different EV subtypes, as well as the signals which enrich proteins in the EVs. 


\section{Lipid Content}

In addition to the proteins within EVs, the lipid composition has been extensively studied in various settings (Van Blitterswijk et al. 1982; Carayon et al. 2011; Llorente et al. 2013). In general, the lipid composition share common features with the cells of origin. Although further investigation has shown that some lipids can be specifically associated with different types of EVs. Lipids enriched in EV include sphingomyelin, cholesterol, ganglioside GM3, disaturated lipids, phosphatidylserine, and ceramide (Llorente et al. 2013). In contrast, phosphatidylcholine and diacyl-glycerol are decreased compared to the lipid membrane composition of the cell of origin (Laulagnier et al. 2004). More examples of lipid enrichment are found in vesicles derived from the MVBs which contain more phosphatidylserine facing the extracellular environment as compared to the cellular plasma membrane, a feature that may facilitate their internalization by recipient cells (Subra et al. 2007; Fitzner et al. 2011). Although the lipid composition of MVs is highly similar to that of their donor cell, vesicles are unique in being enriched for polyunsaturated glycerophosphoserine and phosphatidylserine (Bicalho et al. 2013; Zaborowski et al. 2015). Overall, the membrane composition of both MVs and exosomes contains more phosphatidylserine as compared to the cellular plasma membrane composition. But the differences in lipid composition between the different types of vesicles reflect the biogenesis of the different types of EVs, either originating from the MVBs or the plasma membrane.

\section{Nucleic Acid Content}

A diverse composition of genetic material is found in EVs. In a small number of cases, DNA has been found, including genomic and mitochondrial DNA (Guescini et al. 2010; Balaj et al. 2011; Waldenström et al. 2012). But overall, EVs are primarily enriched with small RNAs, with many derived from ribosomal $18 \mathrm{~S}$ and $28 \mathrm{~S}$ rRNAs and tRNAs. Using various techniques, including next-generation sequencing, an abundance of small RNAs have been characterized. In addition to the commonly known RNA species, such as mRNAs, miRNAs, and rRNAs, long and short non-coding RNA, tRNA fragments, piwi-interacting RNA, vault RNA, and Y RNA have been found in EVs (Crescitelli et al. 2013; Cheng et al. 2013; Huang et al. 2013; Ogawa et al. 2013; Xiao et al. 2012; Nolte'T Hoen et al. 2012; Li et al. 2013) (Fig. 3a). Most of the RNA in EVs is around 200 nucleotides in length with a much smaller portion extending out to $4 \mathrm{~kb}$ (Batagov and Kurochkin 2013). So, although there appear to be some intact mRNA and long ncRNAs, most are probably fragmented, both in exosomes and MVs (Fig. 3a, b). Interestingly, circular RNAs are also enriched and stable in EVs ( $\mathrm{Li}$ et al. 2015). Packaging of RNA within the lipid bilayer membrane is thought to protect it from RNase digestion once released into the extracellular environment (Fig. 3b). Alternatively, different RNA species can also be stably associated with ribonucleoproteins (RNPs), such as argonaute 2 (AGO2), or high- and low-density lipoproteins (HDLs and LDLs), which can be associated with the EVs or included with the EV fraction depending on the isolation procedure (Arroyo et al. 2011; Vickers et al. 2011; Vickers and Remaley 2012).

\section{How Does RNA Get Packaged into EVs?}

One of the surprising aspects of EV content has been that it has a somewhat different profile as compared to the RNA content of the cells from which it is derived (Skog et al. 2008; Pigati et al. 2010; Guduric-Fuchs et al. 2012; Jenjaroenpun et al. 2013). Since the discovery that specific RNAs are enriched in EVs, the search for selective loading mechanisms has been underway (Fig. 3c). In general, the RNA cargo of the EVs reflects the levels and types of cytoplasmic content, and is based on the biogenesis of the EVs and type and physiologic state of the cells releasing them. As such, it has been shown that miRNA are sorted to EVs by cell-activation-dependent changes of miRNA target levels in the cell of origin. This was found by overexpressing miRNA target sequences, resulting in relative miRNA enrichment in P-bodies and depletion from MVBs and EVs. Conversely, overexpression of a miRNA enriches it in EVs (de Jong et al. 2012; Squadrito et al. 2014). In depth analysis of the contents of EVs shows that specific mRNA fragments are enriched, especially the $3^{\prime} \mathrm{UTR}$ fragment of mRNAs (Batagov and Kurochkin 2013). In addition, specific sequences were found to be associated with loading into EVs. For example, a sequence motif within the $3^{\prime}$ UTR of a number of mRNAs enriched in EVs may act as a "zipcode" that targets mRNAs into EVs. One potential zipcode consists of a 25 nucleotide sequence which contains a short CTGCC core domain on a stemloop structure and carries a miR-1289 binding site (Bolukbasi et al. 2012). Interaction with this miRNA with the zipcode increases loading of RNAs containing this sequence.

Different mechanisms have been proposed for the cargo selection of miRNAs into EVs. First, a four nucleotide motif (GGAG) has been found to be enriched in miRNAs in exosomes and an interaction between this motif and the ribonucleoprotein (hnRNPA2B1) appears to be involved in loading these miRNAs into MVBs (Villarroya-Beltri et al. 2013). This RNP is also involved in the RNA transport in oligodendrocytes and must undergo a post-translational 

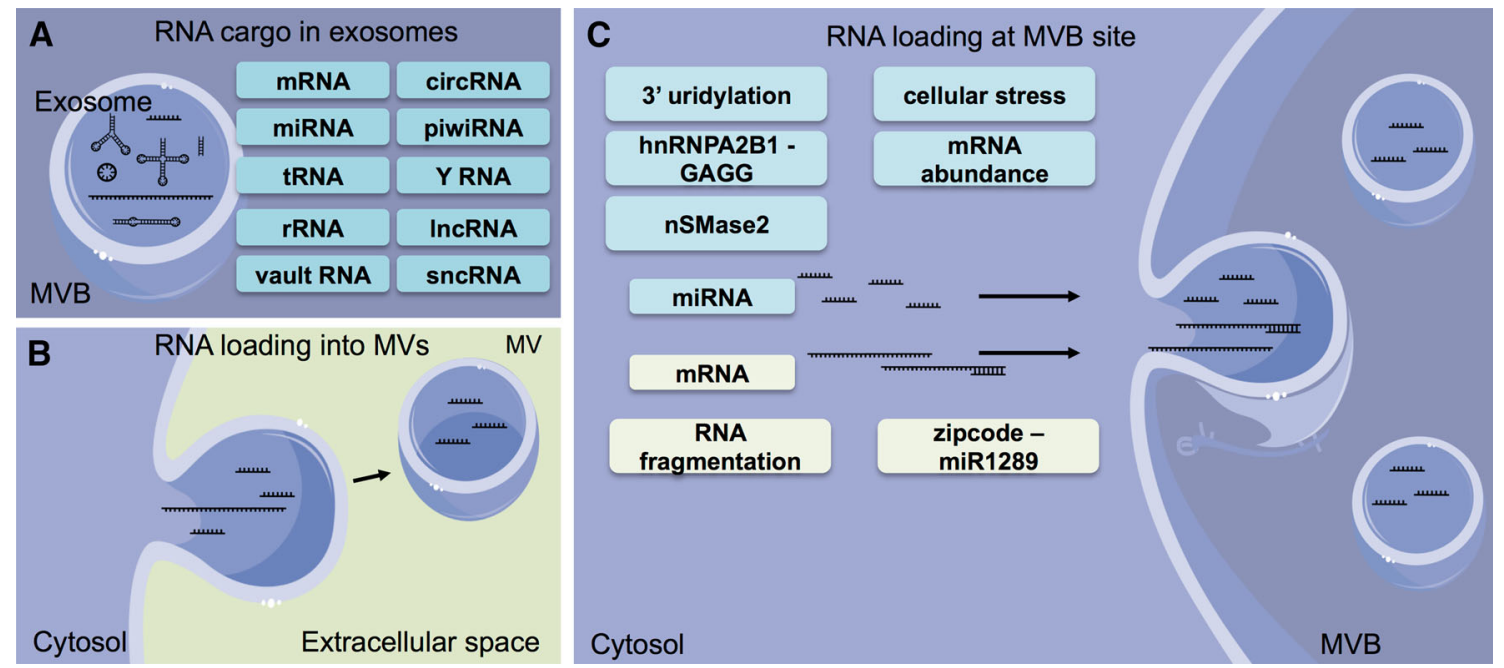

Fig. 3 RNA loading mechanisms and RNA species found in EVs. a A graphical representation of the different RNA species found in EVs including mRNA, miRNA, tRNA, rRNA, vault RNA, circRNA, Y RNA, lncRNA, and sncRNA. b Packaging of RNA within the lipid bilayer membrane is thought to protect it from RNase digestion once released into the extracellular environment. c Different mechanisms of mRNA and miRNA loading into EV as shown in the left panel include: the enrichment of miRNA in EVs due to cellular stress;

modification (sumoylation) prior to loading of miRNAs into EVs (Munro et al. 1999). Notably, miR-1289, the binding site for which is found in the zipcode for mRNA (Bolukbasi et al. 2012), contains the hexanucleotide motif found to be enriched in the miRNAs present in EVs (Villarroya-Beltri et al. 2013). Second, posttranscriptional modifications of miRNAs, in the form of $3^{\prime}$ end uridylation, appears to contribute to direct miRNA sorting into EVs whereas $3^{\prime}$-end adenylated miRNA isoforms are relatively enriched in cells (Koppers-Lalic et al. 2014). Third, overexpression of nSMase 2 increased extracellular amounts of miRNAs. Since this protein is also involved in MVB biogenesis, this increase in miRNA in EVs could be due to an increase in exosome production (Kosaka et al. 2010). Fourth, AGO2, a protein associated with the RISC complex involved in RNA silencing, is thought to control the loading of miRNA into EVs (Guduric-Fuchs et al. 2012) and RNPs (Arroyo et al. 2011). Knockout of AGO2 decreases the amount of specific miRNAs in EVs, which are normally enriched in this fraction. Although the role of AGO2 in miRNA loading remains unclear, some studies report the absence of this protein in exosomes, but only found localized at the site of MVBs and endosomes inside cells (Gibbings et al. 2009), while others report AGO2 to be present in EVs ( $\mathrm{Li}$ et al. 2012) (Fig. 3c). The variations on the loading mechanisms of mRNA and miRNA show the complexity of EV research and the probable differences among EV content and vesicle subtypes among cells.
hnRNPA2B1 binding to GAGG motif present miRNA; $3^{\prime}$ end uridylation of miRNAs/increasing nSMase 2 activity resulting in miRNA loading; the abundance of miRNA target mRNA transcripts in the cell and the binding of miRNA to lipids associated with EVs. Loading of mRNA or mRNA fragments is based on the presence of zipcode sequence and association with miR1289. [Components in image derived from Servier Medical Art Powerpoint image bank (Servier 2016)]

\section{Uptake of EVs}

So far, it has been proposed that the cells internalize EVs either by fusion with the plasma membrane or via endocytosis (Mulcahy et al. 2014). Uptake via endocytosis can be categorized into the different types of endocytotic processes, including clathrin-mediated endocytosis, caveolinmediated endocytosis, lipid raft-mediated endocytosis, macropinocytosis, and phagocytosis. The uptake mode of EVs may be dependent on the type of cell and its physiologic state, and whether ligands on the surface of the EV recognize receptors on the surface of the cell or vice versa. Different mechanisms of internalization have been described for different cell types. For example, clathrin-dependent endocytosis or phagocytosis in neurons, macropinocytosis by microglia, phagocytosis or receptormediated endocytosis by dendritic cells, caveolin-mediated endocytosis in epithelial cells, and cholesterol- and lipid raft-dependent endocytosis in tumor cells (Morelli et al. 2004; Barrès et al. 2010; Feng et al. 2010; Fitzner et al. 2011; Montecalvo et al. 2012; Frühbeis et al. 2013; Nanbo et al. 2013; Svensson et al. 2013). An important factor in EV uptake is heparin sulfate proteoglycans (HSPGs) of the plasma membrane of the recipient cell as blocking this with heparin reduces the uptake of EVs in cell culture (Atai et al. 2013; Christianson et al. 2013). Decreased EV uptake was also achieved by blocking the scavenger receptor type B-1 (SR-B1) with a synthetic nanoparticle mimic of HDL 
(HDL NP) that binds SR-B1, resulting in the removal of cholesterol (Plebanek et al. 2015). Lastly, membrane fusion of EVs with the plasma membranes could serve as method of uptake; this requires low $\mathrm{pH}$ conditions which are found within tumors. The cellular uptake and cytosolic release of EV contents is enhanced by combining a $\mathrm{pH}$-sensitive fusogenic peptide to promote the fusion of endosomal and EV membranes inside cells, thus releasing the EV content into the cytosol (Parolini et al. 2009; Nakase and Futaki 2015).

The mode of EV interaction with and/or entry into cells determines their functional effects. The EV membrane surface can trigger signaling through interaction with receptors/ligands on the cell surface without EV entry as, for example with EGFRvIII (Al-Nedawi et al. 2008); Notch and RHEB (Patel et al. 2015); and IFN- $\gamma /$ STAT (Cossetti et al. 2014). In many cases functionality of the EV contents depends on entry into the cytoplasm, and potentially even into the nucleus. Direct entry into the cytoplasm can be achieved by fusion of EVs to the plasma membrane of the recipient cells, but some form or endocytosis seems to be the most common mode of entry (Mulcahy et al. 2014). If the EVs enter by endocytosis, their cargo must exit that inherently degradative pathway, as endosomes mature into lysosomes, or be ejected out again through the MVBplasma membrane fusion pathway. There must be a way through this maze, as so far, the functional transfer of nucleic acids has been described both in culture as well as in vivo (e.g., Pegtel et al. 2010; Ridder et al. 2014; Lai et al. 2015). The mechanism of effective transfer out of the endosomal compartment is still unclear. This process has been visualized using fluorescent probes labeling EVs in tumor and dendritic cells (Parolini et al. 2009; Montecalvo et al. 2012). A different approach utilized luciferin-loaded EVs internalization into cytosol containing luciferase which allowed monitoring of the fate of the cargo (Abrami et al. 2013). To conclude, different cell types are able to take up EV using various mechanisms resulting in either functional transfer of cargo or degradation of the EV content. The fate may be determined by cell-specific ligands/receptors that "direct the conversation."

\section{Conclusion}

The field of EVs is expanding rapidly. Although a lot of new exciting findings and applications for EVs are being published, the need for general consensus on the mode of isolation, classification and contents of different EV subtypes remains to be determined. By developing more selective isolation techniques, it should be possible to distinguish between the different subpopulations of vesicles and define their biogenesis, cargo, and function more precisely. Once accomplished, future research can focus on new methods to manipulate the biogenesis, content loading, composition, release, and interaction as a means not only for understanding the "language" of EVs, but also for the development of novel therapeutic strategies.

Acknowledgments We thank Suzanne McDavitt for skilled editorial assistance. This work was supported by the NIH Common Fund through the Office of Strategic Coordination/Office of the NIH Director, NCI U19 CA179563 and by NIH/NCI P01 CA069246 (XOB).

\section{Compliance with ethical standards}

Conflict of interest The authors declared that there is no conflict of interest.

\section{References}

Abrami L, Brandi L, Moayeri M et al (2013) Hijacking multivesicular bodies enables long-term and exosome-mediated long-distance action of anthrax toxin. Cell Rep 5:986-996. doi:10.1016/j. celrep.2013.10.019

Akers JC, Gonda D, Kim R et al (2013) Biogenesis of extracellular vesicles (EV): exosomes, microvesicles, retrovirus-like vesicles, and apoptotic bodies. J Neurooncol 113:1-11

Al-Nedawi K, Meehan B, Micallef J et al (2008) Intercellular transfer of the oncogenic receptor EGFRvIII by microvesicles derived from tumour cells. Nat Cell Biol 10:619-624. doi:10.1038/ ncb 1725

Alonso R, Mazzeo C, Mérida I, Izquierdo M (2007) A new role of diacylglycerol kinase $\alpha$ on the secretion of lethal exosomes bearing Fas ligand during activation-induced cell death of $\mathrm{T}$ lymphocytes. Biochimie 89:213-221. doi:10.1016/j.biochi.2006. 07.018

Alonso R, Mazzeo C, Rodriguez MC et al (2011) Diacylglycerol kinase $\alpha$ regulates the formation and polarisation of mature multivesicular bodies involved in the secretion of Fas ligandcontaining exosomes in $\mathrm{T}$ lymphocytes. Cell Death Differ 18:1161-1173. doi:10.1038/cdd.2010.184

Arroyo JD, Chevillet JR, Kroh EM et al (2011) Argonaute2 complexes carry a population of circulating microRNAs independent of vesicles in human plasma. Proc Natl Acad Sci U S A 108:5003-5008. doi:10.1073/pnas.1019055108

Atai NA, Balaj L, Van Veen H et al (2013) Heparin blocks transfer of extracellular vesicles between donor and recipient cells. J Neurooncol 115:343-351. doi:10.1007/s11060-013-1235-y

Babst M, Katzmann DJ, Estepa-Sabal EJ et al (2002) ESCRT-III: an endosome-associated heterooligomeric protein complex required for MVB sorting. Dev Cell 3:271-282. doi:10.1016/S15345807(02)00220-4

Bache KG, Brech A, Mehlum A, Stenmark H (2003) Hrs regulates multivesicular body formation via ESCRT recruitment to endosomes. J Cell Biol 162:435-442. doi:10.1083/jcb. 200302131

Baietti MF, Zhang Z, Mortier E et al (2012) Syndecan-synteninALIX regulates the biogenesis of exosomes. Nat Cell Biol 14:677-685

Baj-Krzyworzeka M, Szatanek R, Wȩglarczyk K et al (2006) Tumour-derived microvesicles carry several surface determinants and mRNA of tumour cells and transfer some of these 
determinants to monocytes. Cancer Immunol Immunother 55:808-818. doi:10.1007/s00262-005-0075-9

Balaj L, Lessard R, Dai L et al (2011) Tumour microvesicles contain retrotransposon elements and amplified oncogene sequences. Nat Commun 2:180. doi:10.1038/ncomms1180

Barrès C, Blanc L, Bette-Bobillo P et al (2010) Galectin-5 is bound onto the surface of rat reticulocyte exosomes and modulates vesicle uptake by macrophages. Blood 115:696-705. doi:10. 1182/blood-2009-07-231449

Barteneva NS, Maltsev N, Vorobjev IA (2013) Microvesicles and intercellular communication in the context of parasitism. Front Cell Infect Microbiol 3:49. doi:10.3389/fcimb.2013.00049

Batagov AO, Kurochkin IV (2013) Exosomes secreted by human cells transport largely mRNA fragments that are enriched in the $3^{\prime}$ untranslated regions. Biol Direct. doi:10.1186/1745-6150-8-12

Beckler M, Higginbotham JN, Franklin JL et al (2013) Proteomic analysis of exosomes from mutant KRAS colon cancer cells identifies intercellular transfer of mutant KRAS. Mol Cell Proteomics 12:343-355. doi:10.1074/mcp.M112.022806

Benedetto A, Liegeois S, Garnier J-M, et al (2006) A novel exosomemediated apical secretion pathway involving the $\mathrm{V0}$-sector of the V-ATPase in C. elegans epidermal cells. Eur Worm Meet

Bicalho B, Holovati JL, Acker JP (2013) Phospholipidomics reveals differences in glycerophosphoserine profiles of hypothermically stored red blood cells and microvesicles. Biochim Biophys Acta 1828:317-326. doi:10.1016/j.bbamem.2012.10.026

Bishop N, Woodman P (2001) TSG101/mammalian VPS23 and mammalian VPS28 interact directly and are recruited to VPS4induced endosomes. J Biol Chem 276:11735-11742. doi:10. 1074/jbc.M009863200

Bolukbasi MF, Mizrak A, Ozdener GB et al (2012) miR-1289 and "Zipcode"-like sequence enrich mrnas in microvesicles. Mol Ther 1:e10

Brouwers JF, Aalberts M, Jansen JWA et al (2013) Distinct lipid compositions of two types of human prostasomes. Proteomics 13:1660-1666. doi:10.1002/pmic.201200348

Bucki R, Bachelot-Loza C, Zachowski A et al (1998) Calcium induces phospholipid redistribution and microvesicle release in human erythrocyte membranes by independent pathways. Biochemistry 37:15383-15391. doi:10.1021/bi9805238

Carayon K, Chaoui K, Ronzier E et al (2011) Proteolipidic composition of exosomes changes during reticulocyte maturation. J Biol Chem 286:34426-34439. doi:10.1074/jbc.M111. 257444

Cheng L, Sun X, Scicluna BJ et al (2013) Characterization and deep sequencing analysis of exosomal and non-exosomal miRNA in human urine. Kidney Int. doi:10.1038/ki.2013.502

Christianson HC, Svensson KJ, van Kuppevelt TH et al (2013) Cancer cell exosomes depend on cell-surface heparan sulfate proteoglycans for their internalization and functional activity. Proc Natl Acad Sci USA 110:17380-17385. doi:10.1073/pnas.1304266110

Cocucci E, Racchetti G, Meldolesi J (2009) Shedding microvesicles: artefacts no more. Trends Cell Biol 19:43-51

Colombo M, Moita C, van Niel G et al (2013) Analysis of ESCRT functions in exosome biogenesis, composition and secretion highlights the heterogeneity of extracellular vesicles. J Cell Sci 126:5553-5565. doi:10.1242/jcs. 128868

Conde-Vancells J, Rodriguez-Suarez E, Embade N et al (2008) Characterization and comprehensive proteome profiling of exosomes secreted by hepatocytes. J Proteome Res 7:5157-5166. doi:10.1021/pr8004887

Cossetti C, Iraci N, Mercer TR et al (2014) Extracellular vesicles from neural stem cells transfer IFN- $\gamma$ via Ifngr1 to activate Stat1 signaling in target cells. Mol Cell 56:193-204. doi:10.1016/j. molcel.2014.08.020
Crescitelli R, Lässer C, Szabó TG et al (2013) Distinct RNA profiles in subpopulations of extracellular vesicles: apoptotic bodies, microvesicles and exosomes. J Extracell vesicles 2:1-10. doi:10. 3402/jev.v2i0.20677

de Jong OG, Verhaar MC, Chen Y, et al (2012) Cellular stress conditions are reflected in the protein and RNA content of endothelial cell-derived exosomes. J Extracell Vesicles

Denzer K, Kleijmeer MJ, Heijnen HF et al (2000) Exosome: from internal vesicle of the multivesicular body to intercellular signaling device. J Cell Sci 113(Pt 19):3365-3374

Fader CM, Sánchez DG, Mestre MB, Colombo MI (2009) TI-VAMP/ VAMP7 and VAMP3/cellubrevin: two v-SNARE proteins involved in specific steps of the autophagy/multivesicular body pathways. Biochim Biophys Acta 1793:1901-1916. doi:10.1016/ j.bbamcr.2009.09.011

Feng D, Zhao WL, Ye YY et al (2010) Cellular internalization of exosomes occurs through phagocytosis. Traffic 11:675-687. doi:10.1111/j.1600-0854.2010.01041.x

Fernandez-Borja M, Wubbolts R, Calafat J et al (1999) Multivesicular body morphogenesis requires phosphatidylinositol 3-kinase activity. Curr Biol 9:55-58. doi:10.1016/S09609822(99)80048-7

Fitzner D, Schnaars M, van Rossum D et al (2011) Selective transfer of exosomes from oligodendrocytes to microglia by macropinocytosis. J Cell Sci 124:447-458. doi:10.1242/jcs. 074088

Frühbeis C, Fröhlich D, Krämer-Albers EM (2012) Emerging roles of exosomes in neuron-glia communication. Front Physiol. doi:10. 3389/fphys.2012.00119

Frühbeis C, Fröhlich D, Kuo WP et al (2013) Neurotransmittertriggered transfer of exosomes mediates oligodendrocyte-neuron communication. PLoS Biol. doi:10.1371/journal.pbio.1001604

Ghossoub R, Lembo F, Rubio A et al (2014) Syntenin-ALIX exosome biogenesis and budding into multivesicular bodies are controlled by ARF6 and PLD2. Nat Commun 5:3477. doi:10.1038/ ncomms 4477

Gibbings DJ, Ciaudo C, Erhardt M, Voinnet O (2009) Multivesicular bodies associate with components of miRNA effector complexes and modulate miRNA activity. Nat Cell Biol 11:1143-1149

Gonzalez-Begne M, Lu B, Han X et al (2009) Proteomic analysis of human parotid gland exosomes by multidimensional protein identification technology (MudPIT). J Proteome Res 8:1304-1314

Gould SJ, Raposo G (2013) As we wait: coping with an imperfect nomenclature for extracellular vesicles. J Extracell Vesicles 2:3-5. doi:10.3402/jev.v2i0.20389

Gould SJ, Booth AM, Hildreth JEK (2003) The Trojan exosome hypothesis. Proc Natl Acad Sci USA 100:10592-10597. doi:10. 1073/pnas. 1831413100

Graner MW, Alzate O, Dechkovskaia AM et al (2009) Proteomic and immunologic analyses of brain tumor exosomes. FASEB J 23:1541-1557. doi:10.1096/fj.08-122184

Grant BD, Donaldson JG (2009) Pathways and mechanisms of endocytic recycling. Nat Rev Mol Cell Biol 10:597-608. doi:10. 1038/nrm2755

Guduric-Fuchs J, O'Connor A, Camp B et al (2012) Selective extracellular vesicle-mediated export of an overlapping set of microRNAs from multiple cell types. BMC Genom 13:357. doi:10.1186/1471-2164-13-357

Guescini M, Genedani S, Stocchi V, Agnati LF (2010) Astrocytes and Glioblastoma cells release exosomes carrying mtDNA. J Neural Transm 117:1-4. doi:10.1007/s00702-009-0288-8

György B, Szabó TG, Pásztói M et al (2011) Membrane vesicles, current state-of-the-art: emerging role of extracellular vesicles. Cell Mol Life Sci 68:2667-2688 
Henderson MC, Azorsa DO (2012) The genomic and proteomic content of cancer cell-derived exosomes. Front Oncol 2:3389

Henne WM, Buchkovich NJ, Emr SD (2011) The ESCRT pathway. Dev Cell 21:77-91

Henne WM, Stenmark H, Emr SD (2013) Molecular mechanisms of the membrane sculpting ESCRT pathway. Cold Spring Harb Perspect Med. doi:10.1101/cshperspect.a016766

Hsu C, Morohashi Y, Yoshimura SI et al (2010) Regulation of exosome secretion by Rab35 and its GTPase-activating proteins TBC1D10A-C. J Cell Biol 189:223-232. doi:10.1083/jcb. 200911018

Huang X, Yuan T, Tschannen M et al (2013) Characterization of human plasma-derived exosomal RNAs by deep sequencing. BMC Genom 14:319. doi:10.1186/1471-2164-14-319

Jenjaroenpun P, Kremenska Y, Nair VM et al (2013) Characterization of RNA in exosomes secreted by human breast cancer cell lines using next-generation sequencing. PeerJ 1:e201. doi:10.7717/ peerj.201

Kalra H, Simpson RJ, Ji H et al (2012) Vesiclepedia: a compendium for extracellular vesicles with continuous community annotation. PLoS Biol. doi:10.1371/journal.pbio. 1001450

Katzmann DJ, Babst M, Emr SD (2001) Ubiquitin-dependent sorting into the multivesicular body pathway requires the function of a conserved endosomal protein sorting complex, ESCRT-I. Cell 106:145-155. doi:10.1016/S0092-8674(01)00434-2

Kim D-K, Kang B, Kim OY et al (2013) EVpedia: an integrated database of high-throughput data for systemic analyses of extracellular vesicles. J Extracell Vesicles 2:1-7. doi:10.3402/ jev.v2i0.20384

Koppers-Lalic D, Hackenberg M, Bijnsdorp IV et al (2014) Nontemplated nucleotide additions distinguish the small RNA composition in cells from exosomes. Cell Reports 8:1649-1658

Kosaka N, Iguchi H, Yoshioka Y et al (2010) Secretory mechanisms and intercellular transfer of microRNAs in living cells. J Biol Chem 285:17442-17452. doi:10.1074/jbc.M110.107821

Lai CP, Kim EY, Badr CE et al (2015) Visualization and tracking of tumour extracellular vesicle delivery and RNA translation using multiplexed reporters. Nat Commun 6:7029. doi:10.1038/ ncomms 8029

Laulagnier K, Grand D, Dujardin A et al (2004) PLD2 is enriched on exosomes and its activity is correlated to the release of exosomes. FEBS Lett 572:11-14. doi:10.1016/j.febslet.2004. 06.082

Li L, Zhu D, Huang L et al (2012) Argonaute 2 complexes selectively protect the circulating micrornas in cell-secreted microvesicles. PLoS One. doi:10.1371/journal.pone.0046957

Li CCY, Eaton SA, Young PE et al (2013) Glioma microvesicles carry selectively packaged coding and noncoding RNAs which alter gene expression in recipient cells. RNA Biol 10:1333-1344. doi:10.4161/rna.25281

Li Y, Zheng Q, Bao C et al (2015) Circular RNA is enriched and stable in exosomes: a promising biomarker for cancer diagnosis. Cell Res. doi:10.1038/cr.2015.82

Llorente A, Skotland T, Sylvänne T et al (2013) Molecular lipidomics of exosomes released by PC-3 prostate cancer cells. Biochim Biophys Acta 1831:1302-1309. doi:10.1016/j.bbalip.2013.04. 011

Logan MR, Lacy P, Odemuyiwa SO et al (2006) A critical role for vesicle-associated membrane protein-7 in exocytosis from human eosinophils and neutrophils. Allergy Eur J Allergy Clin Immunol 61:777-784. doi:10.1111/j.1398-9995.2006.01089.x

Lötvall J, Hill AF, Hochberg F et al (2014) Minimal experimental requirements for definition of extracellular vesicles and their functions: a position statement from the International Society for Extracellular Vesicles. J Extracell Vesicles 3:26913. doi:10. 3402/jev.v3.26913
Luga V, Zhang L, Viloria-Petit AM et al (2012) Exosomes mediate stromal mobilization of autocrine Wnt-PCP signaling in breast cancer cell migration. Cell 151:1542-1556. doi:10.1016/j.cell. 2012.11.024

Marcilla A, Trelis M, Cortés A et al (2012) Extracellular vesicles from parasitic helminths contain specific excretory/secretory proteins and are internalized in intestinal host cells. PLoS One. doi:10.1371/journal.pone.0045974

Mathivanan S, Simpson RJ (2009) ExoCarta: a compendium of exosomal proteins and RNA. Proteomics 9:4997-5000. doi:10. 1002/pmic.200900351

Mathivanan S, Fahner CJ, Reid GE, Simpson RJ (2012) ExoCarta 2012: database of exosomal proteins RNA and lipids. Nucleic Acids Res. doi:10.1093/nar/gkr828

Matsuo H, Chevallier J, Mayran N et al (2004) Role of LBPA and Alix in multivesicular liposome formation and endosome organization. Science 303:531-534. doi:10.1126/science. 1092425

McCullough J, Fisher RD, Whitby FG et al (2008) ALIX-CHMP4 interactions in the human ESCRT pathway. Proc Natl Acad Sci USA 105:7687-7691. doi:10.1073/pnas.0801567105

Minciacchi VR, Freeman MR, Di Vizio D (2015) Extracellular vesicles in cancer: exosomes, microvesicles and the emerging role of large oncosomes. Semin Cell Dev Biol 40:41-51

Momen-Heravi F, Balaj L, Alian S et al (2012) Alternative methods for characterization of extracellular vesicles. Front Physiol $3: 3389$

Montecalvo A, Larregina AT, Shufesky WJ et al (2012) Mechanism of transfer of functional microRNAs between mouse dendritic cells via exosomes. Blood 119:756-766. doi:10.1182/blood2011-02-338004

Morelli AE, Larregina AT, Shufesky WJ et al (2004) Endocytosis, intracellular sorting, and processing of exosomes by dendritic cells. Blood 104:3257-3266. doi:10.1182/blood-2004-03-0824

Mulcahy LA, Pink RC, Carter DRF (2014) Routes and mechanisms of extracellular vesicle uptake. J Extracell Vesicles 3:1-14. doi:10. 3402/jev.v3.24641

Munro TP, Magee RJ, Kidd GJ et al (1999) Mutational analysis of a heterogeneous nuclear ribonucleoprotein A2 response element for RNA trafficking. J Biol Chem 274:34389-34395. doi:10. 1074/jbc.274.48.34389

Muralidharan-Chari V, Clancy J, Plou C et al (2009) ARF6-regulated shedding of tumor cell-derived plasma membrane microvesicles. Curr Biol 19:1875-1885. doi:10.1016/j.cub.2009.09.059

Nabhan JF, Hu R, Oh RS et al (2012) Formation and release of arrestin domain-containing protein 1-mediated microvesicles (ARMMs) at plasma membrane by recruitment of TSG101 protein. Proc Natl Acad, Sci

Nakase I, Futaki S (2015) Combined treatment with a pH-sensitive fusogenic peptide and cationic lipids achieves enhanced cytosolic delivery of exosomes. Sci Rep 5:10112. doi:10.1038/ srep10112

Nanbo A, Kawanishi E, Yoshida R, Yoshiyama H (2013) Exosomes derived from Epstein-Barr virus-infected cells are internalized via caveola-dependent endocytosis and promote phenotypic modulation in target cells. J Virol 87:10334-10347. doi:10. 1128/JVI.01310-13

Nolte'T Hoen ENM, Buermans HPJ, Waasdorp M et al (2012) Deep sequencing of RNA from immune cell-derived vesicles uncovers the selective incorporation of small non-coding RNA biotypes with potential regulatory functions. Nucleic Acids Res 40:9272-9285. doi:10.1093/nar/gks658

Ogawa Y, Taketomi Y, Murakami M et al (2013) Small RNA transcriptomes of two types of exosomes in human whole saliva determined by next generation sequencing. Biol Pharm Bull 36:66-75. doi:10.1248/bpb.b12-00607 
Ostrowski M, Carmo NB, Krumeich S et al (2010) Rab27a and Rab27b control different steps of the exosome secretion pathway. Nat Cell Biol 12:19-30. doi:10.1038/ncb2000

Pan BT, Johnstone RM (1983) Fate of the transferrin receptor during maturation of sheep reticulocytes in vitro: selective externalization of the receptor. Cell 33(3):967-978

Parolini I, Federici C, Raggi C et al (2009) Microenvironmental pH is a key factor for exosome traffic in tumor cells. J Biol Chem 284:34211-34222. doi:10.1074/jbc.M109.041152

Pasquet JM, Dachary-Prigent J, Nurden AT (1996) Calcium influx is a determining factor of calpain activation and microparticle formation in platelets. Eur J Biochem 239:647-654

Patel B, Patel J, Cho J-H et al (2015) Exosomes mediate the acquisition of the disease phenotypes by cells with normal genome in tuberous sclerosis complex. Oncogene. doi:10.1038/onc.2015.358

Pegtel DM, Cosmopoulos K, Thorley-Lawson DA et al (2010) Functional delivery of viral miRNAs via exosomes. Proc Natl Acad Sci USA 107:6328-6333. doi:10.1073/pnas.0914843107

Pigati L, Yaddanapudi SCS, Iyengar R et al (2010) Selective release of MicroRNA species from normal and malignant mammary epithelial cells. PLoS One 5:e13515

Plebanek MP, Mutharasan RK, Volpert O et al (2015) Nanoparticle targeting and cholesterol flux through scavenger receptor type B-1 inhibits cellular exosome uptake. Sci Rep 5:15724. doi:10. 1038/srep15724

Pols MS, Klumperman J (2009) Trafficking and function of the tetraspanin CD63. Exp Cell Res 315:1584-1592

Puri N, Roche PA (2008) Mast cells possess distinct secretory granule subsets whose exocytosis is regulated by different SNARE isoforms. Proc Natl Acad Sci USA 105:2580-2585. doi:10.1073/ pnas.0707854105

Raiborg C, Stenmark H (2009) The ESCRT machinery in endosomal sorting of ubiquitylated membrane proteins. Nature 458:445452. doi:10.1038/nature07961

Rao SK, Huynh C, Proux-Gillardeaux V et al (2004) Identification of SNAREs involved in synaptotagmin VII-regulated lysosomal exocytosis. J Biol Chem 279:20471-20479. doi:10.1074/jbc. M400798200

Raposo G, Stoorvogel W (2013) Extracellular vesicles: exosomes, microvesicles, and friends. J Cell Biol 200:373-383

Ratajczak J, Miekus K, Kucia M et al (2006) Embryonic stem cellderived microvesicles reprogram hematopoietic progenitors: evidence for horizontal transfer of mRNA and protein delivery. Leukemia 20:847-856. doi:10.1038/sj.leu.2404132

Razi M, Futter CE (2006) Distinct roles for Tsg101 and Hrs in multivesicular body formation and inward vesiculation. Mol Biol Cell 17:3469-3483. doi:10.1091/mbc.E05-11-1054

Regev-Rudzki N, Wilson DW, Carvalho TG et al (2013) Cell-cell communication between malaria-infected red blood cells via exosome-like vesicles. Cell 153:1120-1133. doi:10.1016/j.cell. 2013.04.029

Ridder K, Keller S, Dams M et al (2014) Extracellular vesiclemediated transfer of genetic information between the hematopoietic system and the brain in response to inflammation. PLoS Biol 12:e1001874

Rilla K, Pasonen-Seppänen S, Deen AJ et al (2013) Hyaluronan production enhances shedding of plasma membrane-derived microvesicles. Exp Cell Res 319:2006-2018. doi:10.1016/j. yexcr.2013.05.021

Rilla K, Siiskonen H, Tammi M, Tammi R (2014) Hyaluronan-coated extracellular vesicles- A novel link between hyaluronan and cancer. Adv Cancer Res 123:121-148. doi:10.1016/B978-0-12800092-2.00005-8

Roucourt B, Meeussen S, Bao J et al (2015) Heparanase activates the syndecan-syntenin-ALIX exosome pathway. Cell Res 25:412-428. doi:10.1038/cr.2015.29
Savina A, Furlán M, Vidal M, Colombo MI (2003) Exosome release is regulated by a calcium-dependent mechanism in K562 cells. J Biol Chem 278:20083-20090. doi:10.1074/jbc.M301642200

Servier LL (2016). http://www.servier.com/Powerpoint-image-bank

Shields SB, Oestreich AJ, Winistorfer S et al (2009) ESCRT ubiquitin-binding domains function cooperatively during MVB cargo sorting. J Cell Biol 185:213-224. doi:10.1083/jcb. 200811130

Simpson RJ, Kalra H, Mathivanan S (2012) ExoCarta as a resource for exosomal research. J Extracell Vesicles. doi:10.3402/jev. v1i0.18374

Skog J, Würdinger T, van Rijn S et al (2008) Glioblastoma microvesicles transport RNA and proteins that promote tumour growth and provide diagnostic biomarkers. Nat Cell Biol 10:1470-1476. doi:10.1038/ncb1800

Squadrito ML, Baer C, Burdet F et al (2014) Endogenous RNAs modulate MicroRNA sorting to exosomes and transfer to acceptor cells. Cell Reports 8:1432-1446

Stenmark H (2009) Rab GTPases as coordinators of vesicle traffic. Nat Rev Mol Cell Biol 10:513-525. doi:10.1038/nrm2728

Stoorvogel W, Strous GJ, Geuze HJ et al (1991) Late endosomes derive from early endosomes by maturation. Cell 65:417-427. doi:10.1016/0092-8674(91)90459-C

Stuffers S, Sem Wegner C, Stenmark H, Brech A (2009) Multivesicular endosome biogenesis in the absence of ESCRTs. Traffic 10:925-937. doi:10.1111/j.1600-0854.2009.00920.x

Subra C, Laulagnier K, Perret B, Record M (2007) Exosome lipidomics unravels lipid sorting at the level of multivesicular bodies. Biochimie 89:205-212

Svensson KJ, Christianson HC, Wittrup A et al (2013) Exosome uptake depends on ERK1/2-heat shock protein 27 signaling and lipid raft-mediated endocytosis negatively regulated by caveolin1. J Biol Chem 288:17713-17724. doi:10.1074/jbc.M112. 445403

Tamai K, Tanaka N, Nakano T et al (2010) Exosome secretion of dendritic cells is regulated by Hrs, an ESCRT-0 protein. Biochem Biophys Res Commun 399:384-390. doi:10.1016/j. bbrc.2010.07.083

Tauro BJ, Greening DW, Mathias RA et al (2012) Comparison of ultracentrifugation, density gradient separation, and immunoaffinity capture methods for isolating human colon cancer cell line LIM1863-derived exosomes. Methods 56:293-304. doi:10.1016/j.ymeth.2012.01.002

Théry C, Boussac M, Véron P et al (2001) Proteomic analysis of dendritic cell-derived exosomes: a secreted subcellular compartment distinct from apoptotic vesicles. J Immunol 166:7309-7318. doi:10.4049/jimmunol.166.12.7309

Théry C, Zitvogel L, Amigorena S (2002) Exosomes: composition, biogenesis and function. Nat Rev Immunol 2:569-579. doi:10. $1038 /$ nri855

Théry C, Ostrowski M, Segura E (2009) Membrane vesicles as conveyors of immune responses. Nat Rev Immunol 9:581-593. doi: $10.1038 / \mathrm{nri} 2567$

Tiwari N, Wang CC, Brochetta C et al (2008) VAMP-8 segregates mast cell-preformed mediator exocytosis from cytokine trafficking pathways. Blood 111:3665-3674. doi:10.1182/blood-200707-103309

Turiák L, Misják P, Szabó TG et al (2011) Proteomic characterization of thymocyte-derived microvesicles and apoptotic bodies in BALB/c mice. J Proteomics 74:2025-2033. doi:10.1016/j.jprot. 2011.05.023

Van Blitterswijk WJ, De Veer G, Krol JH, Emmelot P (1982) Comparative lipid analysis of purified plasma membranes and shed extracellular membrane vesicles from normal murine thymocytes and leukemic GRSL cells. Biochim Biophys Acta 688:495-504. doi:10.1016/0005-2736(82)90361-3 
Vickers KC, Remaley AT (2012) Lipid-based carriers of microRNAs and intercellular communication. Curr Opin Lipidol 23:91-97

Vickers KC, Palmisano BT, Shoucri BM et al (2011) MicroRNAs are transported in plasma and delivered to recipient cells by highdensity lipoproteins. Nat Cell Biol 13:423-433. doi:10.1038/ ncb2210

Villarroya-Beltri C, Gutiérrez-Vázquez C, Sánchez-Cabo F et al (2013) Sumoylated hnRNPA2B1 controls the sorting of miRNAs into exosomes through binding to specific motifs. Nat Commun 4:2980. doi:10.1038/ncomms3980

Waldenström A, Gennebäck N, Hellman U, Ronquist G (2012) Cardiomyocyte microvesicles contain DNA/RNA and convey biological messages to target cells. PLoS One. doi:10.1371/ journal.pone. 0034653

Wang T, Gilkes DM, Takano N et al (2014) Hypoxia-inducible factors and RAB22A mediate formation of microvesicles that stimulate breast cancer invasion and metastasis. Proc Natl Acad Sci USA 111:E3234-E3242. doi:10.1073/pnas.1410041111

Witwer KW, Buzás EI, Bemis LT et al (2013) Standardization of sample collection, isolation and analysis methods in extracellular vesicle research. J Extracell Vesicles 2:1-25. doi:10.3402/jev. v2i 0.20360

Wollert T, Hurley JH (2010) Molecular mechanism of multivesicular body biogenesis by ESCRT complexes. Nature 464:864-869. doi:10.1038/nature08849

Wubbolts R, Leckie RS, Veenhuizen PTM et al (2003) Proteomic and biochemical analyses of human $\mathrm{B}$ cell-derived exosomes: potential implications for their function and multivesicular body formation. J Biol Chem 278:10963-10972. doi:10.1074/jbc. M207550200

Xiao D, Ohlendorf J, Chen $\mathrm{Y}$ et al (2012) Identifying mRNA, microrna and protein profiles of melanoma exosomes. PLoS One. doi:10.1371/journal.pone.0046874

Yang J-M, Gould SJ (2013) The cis-acting signals that target proteins to exosomes and microvesicles. Biochem Soc Trans 41:277-282. doi:10.1042/BST20120275

Zaborowski MP, Balaj L, Breakefield XO, Lai CP (2015) Extracellular vesicles: composition, biological relevance, and methods of study. Bioscience 65:783-797. doi:10.1093/biosci/biv084 